\title{
A single copy integration vector that integrates at an engineered site on the Staphylococcus aureus chromosome
}

\author{
Mei G Lei, David Cue, Jimena Alba, Jennifer Junecko, Justin W Graham and Chia Y Lee*
}

\begin{abstract}
Background: Single-copy integration vectors based upon the site-specific recombination systems of bacteriophage are invaluable tools in the study of bacterial pathogenesis. The utility of such vectors is often limited, however, by the fact that integration often results in the inactivation of bacterial genes or has undesirable effects on gene transcription. The aim of this study is to develop an integration vector that does not have a detectable effect on gene transcription upon integration.

Findings: We have developed a single-copy integration system that enables the cloning vector to integrate at a specific engineered site, within an untranscribed intergenic region, in the chromosome of Staphylococcus aureus. This system is based on the lysogenic phage L54a site-specific recombination system in which the L54a phage (attP) and chromosome (attB) attachment sites, which share an 18-bp identical core sequence, were modified with identical mutations. The integration vector, pLL102, was constructed to contain the modified L54a attP site (attP2) that was altered at 5 nucleotide positions within the core sequence. In the recipient strain, the similarly modified attB site (attB2) was inserted in an intergenic region devoid of detectable transcription read-through. Integration of the vector, which is unable to replicate in S. aureus extrachromosomally, was achieved by providing the L54a integrase gene in a plasmid in the recipient. We showed that pLL102 integrated specifically at the engineered site rather than at the native L54a attB site and that integration did not have a significant effect on transcription of genes immediately upstream or downstream of the integration site.
\end{abstract}

Conclusions: In this work, we describe an E. coli-S. aureus shuttle vector that can be used to introduce any cloned gene into the $S$. aureus chromosome at a select site without affecting gene expression. The vector should be useful for genetic manipulation of S. aureus and for marking strains for in vivo studies.

\section{Background}

The ability to genetically manipulate bacterial pathogens is essential for the advancement of research on the mechanism of pathogenesis. This holds true for Staphylococcus aureus, an important human pathogen that has become one of the most serious infectious agents worldwide [1]. One of the most common genetic manipulations is the complementation test, which is typically carried out using plasmid vectors. In $S$. aureus, most plasmid vectors are multi-copy in nature which could result in overexpression of the complementing gene or

\footnotetext{
* Correspondence: clee2@uams.edu

Department of Microbiology and Immunology, University of Arkansas for Medical Sciences, 4301 W. Markham Street, Slot 511, Little Rock, AR 72205, USA
}

(c) 2011 Lei et al; licensee BioMed Central Ltd. This is an Open Access article distributed under the terms of the Creative Commons Attribution License (http://creativecommons.org/licenses/by/2.0), which permits unrestricted use, distribution, and reproduction in any medium, provided the original work is properly cited. could place a heavy metabolic burden on the host bacterium. In order to circumvent these and other problems, we previously developed $S$. aureus vectors that allow cloning of genes in single copy $[2,3]$. These plasmid vectors are based on the site-specific recombination systems of the lysogenic bacteriophage L54a and Ø11. The vectors can not replicate in S. aureus, but because they each carry a bacteriophage attP site, they can integrate into the bacterial chromosome at the attB site for L54a or Ø11, respectively, in the presence of the bacteriophage integrase (Int) $[2,3]$.

The L54a site-specific recombination system is similar to that of coliphage $\lambda$. $\lambda$ site-specific recombination is mediated by a tyrosine recombinase known as integrase, or Int, protein [4]. In coliphage $\lambda$, attP, which is much

\section{Biomed Central}


larger than $a t t B$, provides binding sites for Int and accessory factors whereas the $a t t B$ site only has a binding site for Int $[5,6] . \lambda$ Int binds to two distinct DNA sites in $a t t P$, the arm-type and core-type sites. In the recombination reaction, the binding of accessory proteins to attP causes DNA bending which allows an Int monomer to bind both the arm site and the half core site simultaneously. During the recombination reaction, a synapsis is formed in which 4 Int monomers bind to 4 attP arm sites, 2 attP half core sites and 2 att $B$ half core sites. The resulting higher order nucleoprotein complex allows the integrase to catalyze the strand exchange between short 7 bp sequences, termed overlap sequences, within the 15 bp core sequence [4,5,7].

In the L54a phage site-specific recombination system, the phage $a t t P$ site and the bacterial $a t t B$ site share an identical 18 bp core sequence [8]. The $a t t B$ site is located near the 5 ' end of the geh gene (SAOUHSC0030), which encodes a glycerol ester hydrolase, and therefore lysogenization by L54a results in a lipase negative phenotype [9]. Inactivation of genes in this manner is known as negative lysogenic conversion [10-12]. We have shown that the L54a attP site is between 228-235 bp in length but the L54a attB site is less than 27 bp [13]. The int-encoded integrase of L54a, like $\lambda$ Int, has a tyrosine residue that is required for sitespecific recombination [9]. Thus, the L54a site-specific recombination system is similar to that of $\lambda$.

In addition to the single copy vectors developed by us, other researchers have developed vectors based on the site-specific recombination systems of $\varnothing 13$ [14], and the staphylococcal pathogenicity island SaPI [15]. All these systems depend on the wild type $a t t B$ site of the respective phage or pathogenicity island. One drawback of using the $S$. aureus phage site-specific recombination systems for construction of single copy vectors is that the attB site of most staphylococcal phage is located within an open reading frame (ORF) of a gene [10-12]. As a result, insertion at an $a t t B$ site would interrupt an $S$. aureus gene. This is often undesirable because it could inactivate a gene with an important biological function. In addition, a number of sRNAs have been found in the intergenic regions of the S. aureus chromosome which may be affected even if a phage integrates within an intergenic region $[16,17]$. For the two phage that were the basis of vectors that we previously developed, the L54a attB site is within the geh gene, whereas the $\varnothing 11$ attB site is within a hypothetical gene that was previously misannotated to be in an intergenic region $[2,18]$. Furthermore, the $a t t B$ site used for these vectors in some strains may already have an integrated prophage or pathogenicity island. This will interfere with the subsequent transduction from the readily transformable $S$ aureus strain RN4220 used as the initial recipient of these vectors. In addition to the site-specific recombination systems mentioned above, a Cre/lox recombination system has also been used as a genetic tool for maker removal in strain construction in $S$. aureus [19].

In this report, we describe the construction of a single-copy integration vector for $S$. aureus based on the L54a site-specific recombination system in which the same mutation was introduced into both attB and attP. The vector can replicate in Escherichia coli, which allows for easy DNA manipulations. In S. aureus, the vector integrates with high fidelity into an engineered $a t t B$ site that is devoid of transcription activity.

\section{Results and discussion}

Identical sequence alteration in L54a attP and attB affects integration specificity

In the $\lambda$ site-specific recombination system, a single bp alteration within the 7 bp overlap sequence, in either $a t t P$ or $a t t B$, drastically reduces the efficiency of recombination. However, the identical change in the partner site restores recombination efficiency $[4,20]$. Based on this, the $\lambda$ site-specific recombination system has been developed for recombinant DNA applications (for example, the GATEWAY ${ }^{\circledR}$ cloning system developed by Invitrogen Life Science Technologies).

Since the staphylococcal phage L54a Int-mediated recombination system closely parallels that of $\lambda$, we hypothesized that a similar alteration in integration specificity could be achieved in the L54a system $[4,20]$. This would allow us to develop a single-copy cloning vector that would integrate at a region of our choice with minimal interference on normal biological function of the cells. To this end, we first evaluated 12 intergenic regions for transcriptional activity by RT-PCR. The intergenic region between SAOUHSC00937 and SAOUHSC00938 (ORF designations are according to $S$. aureus strain NCTC8325 annotation) was initially selected for insertion of a mutated attB site. We detected very little transcriptional activity in this region in strain 8325-4 cultures grown for $2 \mathrm{~h}, 4 \mathrm{~h}$ or $16 \mathrm{~h}$ (data not shown), although two sRNAs are known to be encoded within this region [17].

We then tested whether identical sequence alterations of L54a attP and attB affect L54a integration specificity. As shown in Figure 1, a pair of 7-bp imperfect inverted repeats, flanking a 3 bp spacer sequence, is located in the L54a attP and attB sites [8]. The inverted repeats could serve as the binding sites for L54a Int reminiscent of $\lambda$ Int core binding half sites. The spacer region is similar to the overlap region of $\lambda$. Accordingly, we altered $2 \mathrm{bps}$ within the core of the attP and attB pair by overlapping PCR. The altered $a t t P$ was named attP1 (Figure 1). The 1617-bp PCR fragment containing attP1 and the adjacent int gene was cloned into pCL52.2 resulting in plasmid pLL3961. 


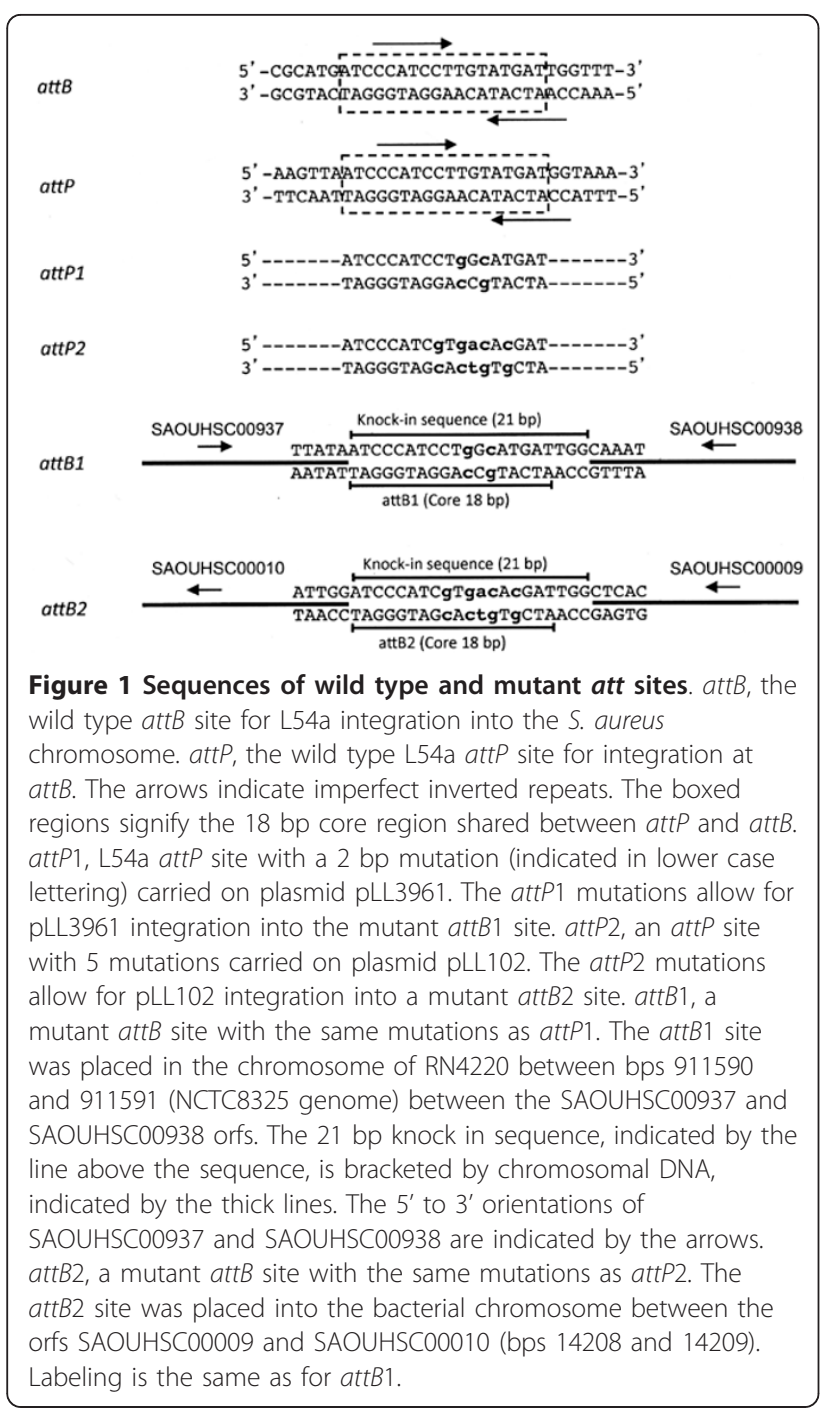

The altered $a t t B$ sequence, named attB1, was introduced into the bacterial chromosome using the pKOR1 system [21] as previously described [22] using RN4220 DNA as template and primers OU937R9, OU937R10, OU937R11 and OU937R12 (Table 1). The 21-bp attB1 fragment was inserted between bp coordinates 911590 and 911591 (S. aureus NCTC8325 genome) of the bacterial chromosome. This is within the intergenic region between SAOUHSC00937 and SAOUHSC00938 of strain RN4220, 29 bp downstream of sRNA RsaF $[17,23]$. The resultant strain, CYL12337, was used as the recipient for integration of pLL3961.

PCR was used in order to determine whether pLL3961 had integrated at $a t t B 1$ or the wild type attB site. Out of 4 integrants that we tested, 3 were integrated at the correct location, attB1 (results not shown). However, in one of the integrants the plasmid had integrated at the wild type L54a attB site in the geh gene. These results indicate that the altered att sites can be recognized by
L54a Int but that the specificity of integration is less than perfect. These results also indicate that the size of the L54a attB site is less than $21 \mathrm{bp}$ in length, which is shorter than the $27 \mathrm{bp}$ that we previously determined [13].

\section{Developing pLL102 with high fidelity integration at an engineered $a t t B$ site}

The above results suggest that phage integration specificity could be altered by introducing the identical bp changes into the core sequence of $a t t P$ and $a t t B$, but 2bp alteration is not enough to ensure high fidelity. Therefore, to increase the specificity, we replaced $5 \mathrm{bp}$ of the att core sequence as shown in Figure 1. The new att site was named attP2. The attP2 fragment was then cloned into pCL25 [2], replacing the wild type L54a attP fragment, to form pLL102 (Figure 2). Since there is still a low level of transcription at the attB1 site described above, we tested additional intergenic regions for insertion of attB2 into the chromosome. We found that transcriptional activity in the region between SAOUHSC00009 and SAOUHSC00010 of the 8325-4 chromosome was undetectable at 3 different time points (after 2, 4 and $16 \mathrm{~h}$ of culture) by RT-PCR (data not shown). The 21 bp attB2 site was then inserted into the intergenic region between $\mathrm{G}$ and $\mathrm{C}$ at coordinates 14208 and 14209 (NCTC8325 genome) of strain RN4220 (Figure 1) by pKOR1 allele replacement. The resultant strain, CYL12348, was confirmed by PCR. Plasmid pYL112 $\Delta 19$, which encodes L54a Int [3], was introduced into CYL12348 to generate strain CYL12349. Plasmid pLL102 was then electroporated into CYL12349. Examination of 22 transformants by PCR (Figure 3) showed that all the transformants carried pLL102 integrated at the attB2 site. None of the transformants had an insertion at the original L54a attB site. These results indicate that pLL102 has a high degree of specificity for the attB2 site. The fact that in the attP2/attB2 pair, the alteration was made in all 3 nucleotides in the spacer region and 2 nucleotides in the mismatched inverted repeat (Figure 1) suggests that these nucleotides are not needed for L54a Int function. These results therefore reaffirm that L54a Int behaves very similarly to that of $\lambda$ Int suggesting conservation of Int functionality across different species as diverse as $E$. coli and $S$. aureus.

\section{Integration of pLL102 at the attB2 site does not affect transcription of the flanking genes}

SAOUHSC00009 (NCTC8325 genome) is annotated as serS which encodes seryl-tRNA synthetase, whereas SAOUHSC00010 is a hypothetical branched chain amino acid transporter. The two genes are transcribed in the same orientation with SAOUHSC00009 being upstream of SAOUHSC00010 (Figure 1). The location 
Table 1 Primers used in this study

\begin{tabular}{|c|c|}
\hline Primer & Sequence \\
\hline L54alnt1 & ggatccacatttagtagctagtactaaaatc \\
\hline L54aAttP1 & ggtttaccatcatgccaggatgggattaacttgtgttaaaaag \\
\hline L54aAttP2 & gttaatcccatcctggcatgatggtaaaccggtcattctct \\
\hline L54aAttP3 & agatctatcgatacggttatatttattcccctac \\
\hline L54aAttP4 & agatctactaaaaggtatctgecctttttctg \\
\hline L54aAttP5 & atcgataatttaggattgtggttatttttgcg \\
\hline L54aAttP6 & ggtttaccatcgtgtcacgatgggattaacttgtgttaaaaag \\
\hline L54aAttP7 & gttaatcccatcgtgacacgatggtaaaccggtcattctct \\
\hline OU937R3 & gaaccaattgaacaagcttgtgaag \\
\hline OU937R8 & ttacttactatttatgaatggccag \\
\hline OU937R9 & gaattcggacaagtttgtacaaaaaagcaggctgtcgctgaagttgcatcaacttgt \\
\hline OU937attBf & ccaaatttataatttgccaatcatgcc \\
\hline OU937attBr & gttcgaaaattataatcccatcctggc \\
\hline OU937R10 & gaaaattataatcccatcctggcatgattggcaaattataaatttggtacataatagac \\
\hline OU937R11 & ataatttgccaatcatgccaggatgggattataattttcgaactggttaaattcg \\
\hline OU937R12 & ggatccggaccactttgtacaagaaagctgggtatccatttagctccgattgcttc \\
\hline OU9R1 & ggggacaagtttgtacaaaaaagcaggctcagcaggtagagatacaagagga \\
\hline OU9R2 & tgcattggatcccatcgtgacacgattggctcactattatatttttacagcac \\
\hline OU9R3 & taatagtgagccaatcgtgtcacgatgggatccaatgcacataacaacaataaattaag \\
\hline OU9R4 & ggggaccactttgtacaagaaagctgggtactaaagttttgagacgaagccac \\
\hline OU9R5 & gtaaaaatataatagtgagccaatcgtgtc \\
\hline OU9R6 & tgtgcattggatcccatcgtgac \\
\hline OU9R7 & atgggtggtaaaacacaaatttc \\
\hline OU9R8 & ctcactattatatttttacagcac \\
\hline OU9R9 & ccaatgcacataacaacaataaattaag \\
\hline OU9R10 & catactacatatcaacgaaatcag \\
\hline SCV1 & gcaacaccacataatggttcac \\
\hline SCV2.1 & tgtgccatgataacagcacg \\
\hline$\overline{S C V 4}$ & acccagtttgtaattccaggag \\
\hline SCV8 & gcacataattgctcacagcca \\
\hline SAO9F3 & aaggtgcgcaattagagcgtgct \\
\hline SAO9R3 & tctgcgttcacaagctgtggtacc \\
\hline SAO10F3 & atgccggtgctgcgcaattt \\
\hline SAO10R3 & ggcgacgccaaa cgtttcgt \\
\hline
\end{tabular}

of the L54a attB2 site in the chromosome was selected because we detected no transcription in this region using RT-PCR. The intergenic region is $649 \mathrm{bp}$ in length and there is a rho-independent terminator close to the 3'end of SAOUHSC00009. In addition, there is no known sRNA in this region $[16,17]$. To avoid interfering with the transcription of the flanking genes, we positioned the attB2 insertion site 31 bp downstream of the terminator and 618 upstream of the SAOUHSC00010 start site ATG. Although our RTPCR experiment did not detect transcripts flanking the insertion site, it is still possible that an artificial insertion at this site could cause alteration in gene expression in the flanking genes. To determine whether the insertion of the attB2 site and the subsequent integration of pLL102 affect transcription of the flanking genes, we performed qRT-PCR of the adjacent genes. We found no significant differences in SAOUHSC00009 or SAOUHSC00010 mRNA levels between RN4220, CYL12349 (i.e., RN4220-attB2) and CYL12376 (i.e., RN4220 attB2(pLL102)) (data not shown) indicating that the insertion of attB2 or the integration of pLL102 at the attB2 site does not affect expression of the surrounding genes. 


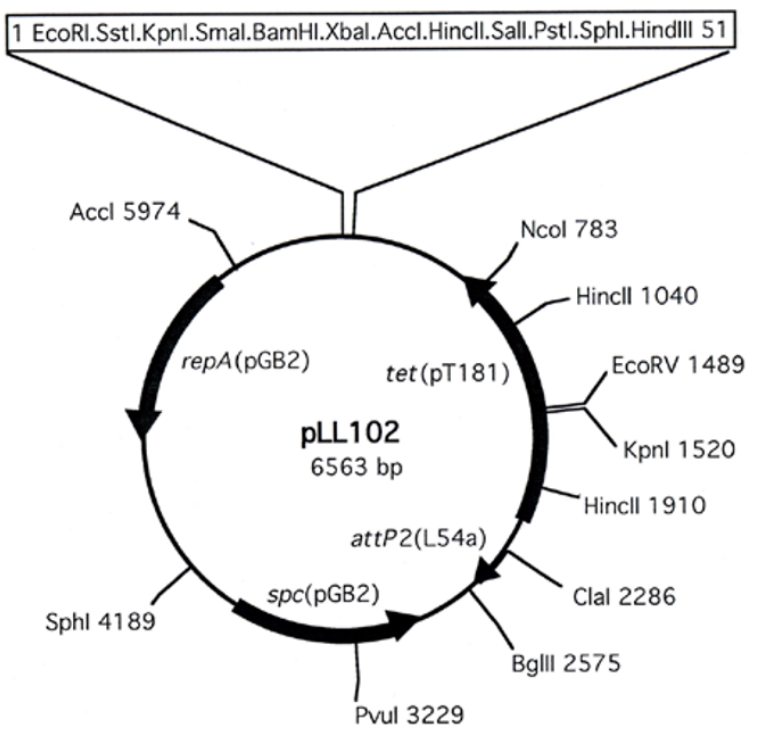

Figure 2 Map of the integration vector pLL102. The spectinomycin resistance gene $\operatorname{spc}(\mathrm{pGB} 2)$ and replication functions $\operatorname{rep} A(p G B 2)$ are both derived from pGB2 [24] and are functional in $E$. coli. The tetracycline resistance gene tet(pT181) is expressed in $S$. aureus. The attP2 site allows for site-specific recombination between attP2 and an engineered attB2 site in the $S$. aureus chromosome. The plasmid is maintained in S. aureus in a single-copy, integrated form. The plasmid sequence has been deposited to GenBank under the accession number JN639000.

\section{Conclusions}

We have developed a single copy integration system that allows insertion of a plasmid vector into the $S$. aureus chromosome at a location devoid of detectable transcription activity. Once a plasmid is inserted into the attB2 site, it can then be moved to any other strain by phage transduction. Since there is no detectable transcription activity at the attB2 site, the insertion is not likely to alter biological function of the clone. Furthermore, the region flanking the attB2 site is highly conserved among all sequenced strains suggesting that moving the integrated plasmid from CYL12349 to other strains is not likely to be hindered by a lack of sequence homology. The system reported here uses a novel, artificial attB site, attB2, thereby avoiding the disadvantages associated with other bacteriophage derived integration vectors. Additionally, the attB2 site is portable and could be inserted into other locations of the chromosome. Thus, our results indicate that plasmid pLL102 is a suitable vector for cloning genes in single copy. In addition, our system can be used to mark a strain with a readily detectable reporter gene, which could be useful for studying pathogenesis in vivo, without affecting gene expression of the marked strains.

\section{Methods}

\section{Bacterial strains, plasmids and culture conditions}

The strains and plasmids used in this study are listed in Table 2. E. coli was grown in Luria broth or Luria agar (Difco). S. aureus was grown in tryptic soy broth (TSB) or TSB agar (Difco). E. coli media were supplemented with $34 \mu \mathrm{g} / \mathrm{ml}$ chloramphenicol, $100 \mu \mathrm{g} / \mathrm{ml}$ penicillin, or $50 \mu \mathrm{g} / \mathrm{ml}$ spectinomycin, as appropriate. S. aureus media were supplemented with $10 \mu \mathrm{g} / \mathrm{ml}$ chloramphenicol, or $3 \mu \mathrm{g} / \mathrm{ml}$ tetracycline as appropriate. Recombinant plasmids were initially constructed in E. coli then electroporated into S. aureus as described [25]. Transduction between $S$. arueus strains was carried out by phage 52A.

\section{RT-PCR and qRT-PCR section}

RNA for RT-PCR and qRT-PCR was isolated as described [22]. RT-PCR was performed using the Quantitech Reverse Transcription Kit from Qiagen Inc., Valencia, CA. RT-PCR using primers OU9R7, OU9R8, OU9R9 and OU9R10 (Table 1) was performed to probe for transcripts in the intergenic region between SAOUHSC00009 and SAOUHSC00010 using RNA isolated from 4, 8 and $18 \mathrm{~h}$ cultures of 8325-4.

To determine whether the insertion of the attB2 site and the subsequent integration of pLL102 affected transcription of the flanking genes, we performed qRT-PCR as described [28] of the adjacent genes using primers SAO9F3 and SAO9R3 for SAOUHSC00009 and SAO10F3 and SAO10F3 for SAOUHSC00010. RNA for qRT-PCR was isolated from exponential phase and overnight cultures of strains RN4220, CYL12349 and CYL12376.

\section{Generation of modified $a t t P$ and $a t t B$}

The attP1 and $a t t B 1$ sites were created by altering two bp within the core of attP and $a t t B$, respectively, by overlapping PCR using primers L54aInt1, L54aAttP1, L54aAttP2 and L54aAttP3 (Table 1), and L54a phage DNA as template. The altered $a t t P$ and $a t t B$ were named $a t t P 1$ and $a t t B 1$, respectively (Figure 1 ). The 1617-bp fragment containing attP1 and the adjacent int gene was cloned into pCL52.2 resulting in plasmid pLL3961. All PCR-amplified fragments were verified by DNA sequencing.

The altered $a t t B$ sequence, named $a t t B 1$, was introduced into the bacterial chromosome by the pKOR1 system as previously described $[21,22]$ using RN4220 DNA as template and primers OU937R9, OU937R10, OU937R11 and OU937R12 (Table 1). The 21-bp attB1 fragment was inserted between bp coordinates 911590 and 911591 ( $S$. aureus NCTC8325 genome) of the bacterial chromosome. This is within the intergenic region between SAOUHSC00937 and SAOUHSC00938 of strain RN4220, 


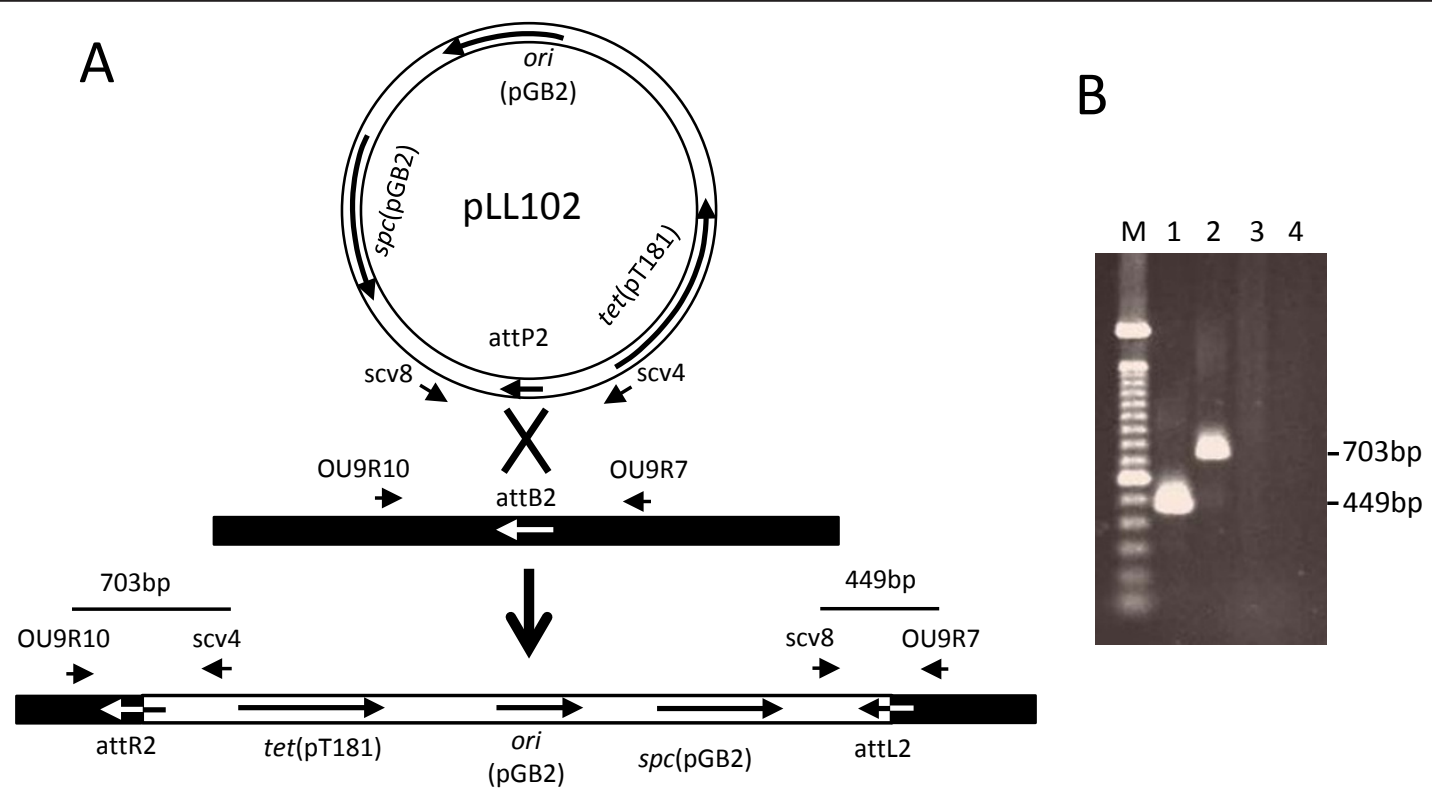

Figure 3 Integration of pLL102 into an engineered attB2 site. Figure 3A illustrates recombination between the attP2 site on pLL102 and the attB2 site in the chromosome of CYL12349. The same 5 bp mutations were introduced into the bacteriophage L54a wild type attP and attB sites to construct attP2 and attB2. The attB2 site was placed in the chromosome (thick solid line) of S. aureus strain RN4220 between the orfs for SAOUHSC00009 (serS) and SAOUHSC00010. Recombination between attP2 and attB2 results in integration of pLL102 into the chromosome as illustrated in the lower portion of the figure. The L54a Int protein is provided in trans by PYL112 $\triangle 19$. Short arrows represent the location of the primers. Figure 3B, Verification of integration via PCR. Genomic DNA was isolated from CYL12376 and used in PCR reactions. Products were analyzed on $1 \%$ agarose gels. Lane 1 shows the product from PCR using primers SCV8 and OU9R7. Lane 2 shows the product from using primer pairs OU9R10 and sCV4. The priming sites and predicted sizes of the PCR products are shown in the lower portion of Figure 3A. Lanes 3 and 4 contain PCR reactions using primer pairs Scv1 and Scv8 and Scv4 and Scv2.1, respectively, to verify that pLL102 did not integrate into the wild type attB site that is flanked by Scv1 and Scv2.1 primer sites (not shown [2]). Integration at the wild type attB site would generate $1.3 \mathrm{~kb}$ and 0.7 $\mathrm{kb}$ fragments in lanes 3 and 4, respectively.

Table 2 Bacterial strains and plasmids used in this study

\begin{tabular}{|c|c|c|}
\hline Strain or Plasmid & Relevant Characteristics & Reference or Source \\
\hline \multicolumn{3}{|l|}{ Staphylococcus aureus } \\
\hline RN4220 & Restriction deficient laboratory strain & [26] \\
\hline CYL12337 & RN4220 attB1 & This study \\
\hline CYL12348 & RN4220 attB2 & This study \\
\hline CYL12349 & CYL12348 (pYL112வ19) & This study \\
\hline CYL12376 & CYL12349 (pLL102) & This study \\
\hline \multicolumn{3}{|l|}{$\overline{\text { E. coli }}$} \\
\hline $\mathrm{DH} 5 \alpha$ & Strain for cloning and plasmid maintenance & Invitrogen \\
\hline XL1-Blue & Strain for cloning and plasmid maintenance & Stratagene \\
\hline \multicolumn{3}{|l|}{ Plasmids } \\
\hline pCL52.2 & Temperature-sensitive cloning vector & [27] \\
\hline pLL3961 & Derivative of pCL52.2 carrying attP1 and the L54a int gene & This study \\
\hline $\mathrm{pCL} 25$ & Carries wild-type attP of L54a & [2] \\
\hline pKOR1 & Used for allele replacement in S. aureus & [21] \\
\hline pYL112 19 & Encodes integrase of phage $\mathrm{L} 54 \mathrm{a}$ & [3] \\
\hline pLL102 & Derivative of $\mathrm{pCL} 25$ carrying attP2 & This study \\
\hline
\end{tabular}


29 bp downstream of sRNA RsaF $[17,23]$. The insertion was verified by PCR using primer pairs OU937attBf/ OU937R9 and OU937attBr/OU937R12. The resultant strain, CYL12337, was used as the recipient for integration of pLL3961. The integration was confirmed by PCR using primer pairs OU937R3/L54aAttP3 and OU937R8/ L54aAttP3. Primer pairs scv2.1/L54aAttP3 and scv1/ L54aAttP4 were used to determine whether a plasmid had integrated at the wild type $a t t B$ site.

The attP and attB sites with 5 bp substitutions were constructed by overlapping PCR using primers L54aAttP4, L54aAttP5, L54aAttP6 and L54aAttP7 (Table 1). The amplified fragments were verified by DNA sequencing. The new att sites were named attP2 and attB2 (Figure 1). The 289-bp attP2 fragment was then cloned into ClaI-BglII-digested pCL25 replacing the 350-bp wild type L54a attP fragment to form pLL102 (Figure 2). The 21-bp attB2 site was then inserted into the intergenic region between $\mathrm{G}$ and $\mathrm{C}$ at coordinates 14208 and 14209 (NCTC8325 genome) of strain RN4220 (Figure 1) using the pKOR1 allele replacement vector and primers OU9R1, OU9R2, OU9R3 and OU9R4. The resultant strain, CYL12348, was confirmed by PCR using primer pairs OU9R1/OU9R6 and OU9R4/OU9R5. Plasmid pYL112 $\Delta 19$, which encodes L54a Int [3], was introduced into CYL12348 by phage 52A transduction and selection for chloramphenicol resistance to generate strain CYL12349. Plasmid pLL102 was then electroporated into CYL12349 and transformants were selected with $3 \mu \mathrm{g} / \mathrm{ml}$ tetracycline.

\section{Acknowledgements \\ This work was supported by grants Al037027 and Al067857 to C. Y. L from the National Institute of Allergy and Infectious Diseases.}

\section{Authors' contributions}

$M G L, D C$ and $C Y L$ designed the research and wrote the manuscript. MGL carried out chromosome site selection experiments. DC carried out gene expression experiments. JA performed technical experiments in plasmid and strain construction. JJ and JWG carried out strain construction and integration experiments. All authors read and approved the final manuscript.

\section{Competing interests}

The authors declare that they have no competing interests.

Received: 9 September 2011 Accepted: 5 January 2012

Published: 5 January 2012

\section{References}

1. David MZ, Daum RS: Community-associated methicillin-resistant Staphylococcus aureus: epidemiology and clinical consequences of an emerging epidemic. Clinical Microbiology Reviews 2010, 23(3):616-687.

2. Luong T, Lee CY: Improved single-copy integration vectors for Staphylococcus aureus. J Microbiol Methods 2007, 70(1):186-190.

3. Lee $\mathrm{CY}$, Buranen $\mathrm{SL}$, Ye ZH: Construction of single-copy integration vectors for Staphylococcus aureus. Gene 1991, 103(1):101-105.

4. Landy A: Dynamic, structural, and regulatory aspects of lambda sitespecific recombination. Annu Rev Biochem 1989, 58:913-949.
5. Radman-Livaja M, Biswas T, Ellenberger T, Landy A, Aihara H: DNA arms do the legwork to ensure the directionality of lambda site-specific recombination. Curr Opin Struct Biol 2006, 16(1):42-50.

6. Thompson JF, de Vargas LM, Skinner SE, Landy A: Protein-protein interactions in a higher-order structure direct lambda site-specific recombination. J Mol Biol 1987, 195(3):481-493.

7. Moitoso de Vargas L, Kim S, Landy A: DNA looping generated by DNA bending protein IHF and the two domains of lambda integrase. Science 1989, 244(4911):1457-1461.

8. Lee CY, landolo Jj: Integration of staphylococcal phage L54a occurs by site-specific recombination: structural analysis of the attachment sites. Proc Natl Acad Sci USA 1986, 83(15):5474-5478.

9. Ye $\mathrm{ZH}$, Lee $\mathrm{CY}$ : Nucleotide sequence and genetic characterization of staphylococcal bacteriophage L54a int and xis genes. J Bacteriol 1989, 171(8):4146-4153.

10. Carroll D, Kehoe MA, Cavanagh D, Coleman DC: Novel organization of the site-specific integration and excision recombination functions of the Staphylococcus aureus serotype $\mathrm{F}$ virulence-converting phages phi 13 and phi 42. Mol Microbiol 1995, 16(5):877-893.

11. Coleman D, Knights J, Russell R, Shanley D, Birkbeck TH, Dougan G, Charles I: Insertional inactivation of the Staphylococcus aureus beta-toxin by bacteriophage phi 13 occurs by site- and orientation-specific integration of the phi 13 genome. Mol Microbiol 1991, 5(4):933-939.

12. Lee $C Y$, landolo JJ: Lysogenic conversion of staphylococcal lipase is caused by insertion of the bacteriophage L54a genome into the lipase structural gene. J Bacteriol 1986, 166(2):385-391.

13. Lee $C Y$, Buranen SL: Extent of the DNA sequence required in integration of staphylococcal bacteriophage L54a. J Bacteriol 1989, 171(3):1652-1657.

14. Mainiero M, Goerke C, Geiger T, Gonser C, Herbert S, Wolz C: Differential target gene activation by the Staphylococcus aureus two-component system saeRS. J Bacteriol 2010, 192(3):613-623.

15. Charpentier E, Anton Al, Barry P, Alfonso B, Fang Y, Novick RP: Novel cassette-based shuttle vector system for gram-positive bacteria. Appl Environ Microbiol 2004, 70(10):6076-6085.

16. Pichon C, Felden B: Small RNA genes expressed from Staphylococcus aureus genomic and pathogenicity islands with specific expression among pathogenic strains. Proc Natl Acad Sci USA 2005, 102(40):14249-14254.

17. Bohn C, Rigoulay C, Chabelskaya S, Sharma CM, Marchais A, Skorski P, Borezee-Durant E, Barbet $R$, Jacquet $E$, Jacq $A$, et al: Experimental discovery of small RNAs in Staphylococcus aureus reveals a riboregulator of central metabolism. Nucleic Acids Res 2010, 38(19):6620-6636.

18. Gillaspy AF, Worrell V, Orvis J, Roe BA, Dyer DW, landolo JJ: The Staphylococcus aureus NCTC 8325 genome. In Gram-positive pathogens 2 Edited by: Fischetti V, Novick RP, Ferretti J, Portnoy D, Rood J. American Society for Microbiology; Washington, DC; 2006:381-412.

19. Leibig M, Krismer B, Kolb M, Friede A, Götz F, Bertram R: Marker removal in staphylococci via Cre recombinase and different lox sites. Appl Environ Microbiol 2008, 74(5):1316-1323.

20. Weisberg RA, Landy A: Site-specific recombination in phage lambda. In Lambda II.. 2 edition. Edited by: Hendrix RW, et al. Cold Spring Harbor, NY: Cold Spring Harbor Press; 1983:211-250.

21. Bae T, Schneewind O: Allelic replacement in Staphylococcus aureus with inducible counter-selection. Plasmid 2006, 55(1):58-63.

22. Lei MG, Cue D, Roux CM, Dunman PM, Lee CY: Rsp inhibits attachment and biofilm formation by repressing $\mathrm{fnbA}$ in Staphylococcus aureus MW2. J Bacteriol 2011, 193(3):686-694.

23. Geissmann T, Chevalier C, Cros MJ, Boisset S, Fechter P, Noirot C, Schrenzel J, Francois P, Vandenesch F, Gaspin C, et al: A search for small noncoding RNAs in Staphylococcus aureus reveals a conserved sequence motif for regulation. Nucleic Acids Res 2009, 37(21):7239-7257.

24. Churchward G, Belin D, Nagamine Y: A pSC101-derived plasmid which shows no sequence homology to other commonly used cloning vectors. Gene 1984, 31(1-3):165-171.

25. Kraemer GR, landolo JJ: High-frequency transformation of Staphylococcus aureus by electroporation. Curr Microbiol 1990, 21:373-376.

26. Kreiswirth BN, Lofdahl S, Betley MJ, O'Reilly M, Schlievert PM, Bergdoll MS Novick RP: The toxic shock syndrome exotoxin structural gene is not detectably transmitted by a prophage. Nature 1983, 305(5936):709-712. 
27. Sau S, Sun J, Lee CY: Molecular characterization and transcriptional analysis of type 8 capsule genes in Staphylococcus aureus. J Bacteriol 1997, 179(5):1614-1621.

28. Luong TT, Dunman PM, Murphy E, Projan SJ, Lee CY: Transcription Profiling of the mgrA Regulon in Staphylococcus aureus. J Bacteriol 2006, 188(5):1899-1910.

doi:10.1186/1756-0500-5-5

Cite this article as: Lei et al: A single copy integration vector that integrates at an engineered site on the Staphylococcus aureus chromosome. BMC Research Notes 2012 5:5.

Submit your next manuscript to BioMed Central and take full advantage of:

- Convenient online submission

- Thorough peer review

- No space constraints or color figure charges

- Immediate publication on acceptance

- Inclusion in PubMed, CAS, Scopus and Google Scholar

- Research which is freely available for redistribution

Submit your manuscript at www.biomedcentral.com/submit 